\title{
Billiards in magnetic fields: A molecular dynamics approach
}

\author{
M. Aichinger, ${ }^{1}$ S. Janecek, ${ }^{2,3}$ and E. Räsänen ${ }^{4,2, *}$ \\ ${ }^{1}$ Johann Radon Institute for Computational and Applied Mathematics (RICAM), \\ Austrian Academy of Sciences, Altenberger Strasse 69, A-4040 Linz, Austria \\ ${ }^{2}$ Institut für Theoretische Physik, Johannes Kepler Universität, A-4040 Linz, Austria \\ ${ }^{3}$ Institut de Ciència de Materials de Barcelona, Campus UAB, 08193 Bellaterra, Barcelona, España. \\ ${ }^{4}$ Nanoscience Center, Department of Physics, University of Jyväskylä, FI-40014 Jyväskylä, Finland
}

(Dated: November 9, 2018)

\begin{abstract}
We present a computational scheme based on classical molecular dynamics to study chaotic billiards in static external magnetic fields. The method allows to treat arbitrary geometries and several interacting particles. We test the scheme for rectangular single-particle billiards in magnetic fields and find a sequence of regularity islands at integer aspect ratios. In the case of two Coulomb-interacting particles the dynamics is dominated by chaotic behavior. However, signatures of quasiperiodicity can be identified at weak interactions, as well as regular trajectories at strong magnetic fields. Our scheme provides a promising tool to monitor the classical limit of many-electron semiconductor nanostructures and transport systems up to high magnetic fields.
\end{abstract}

PACS numbers: 05.45.Pq,82.40.Bj,73.21.La

\section{INTRODUCTION}

Classical and quantum billiard systems [1, 2] are of significant interest both in nonlinear physics and in applications based on low-dimensional nanostructures [3]. For example, quasi-two-dimensional (quasi-2D) quantum dots [4] are studied in view of emerging applications in the field of quantum computation [5]. They exhibit deterministic ballistic motion of the electrons as "billiard balls" and provide the possibility to tune their shape, size, and electron number. A particularly intriguing feature is the connection between classical dynamics and the statistical properties of the corresponding quantum system [6, 7]. For systems with mixed chaotic and regular dynamics, the Berry-Robnik formula [8] links the volume ratio of regular and chaotic regions in classical phase space to the quantum-mechanical level distribution [9, 10].

External magnetic fields pose, on the one hand, an interesting complication to classical (and quantum) billiards [11], and, on the other hand, provide an easily accessible way to experimentally control the particle dynamics. Recently, magnetic fields have been used to manipulate electron transport in coupled electron billiards 12]. In many cases, e.g., in rectangular 13, 14, 15, 16] or triangular [17] billiards, an external magnetic field leads to mixed dynamics between regularity and chaoticity. The breaking of time reversal symmetry due to the presence of a magnetic field results in new properties of the level spacing statistics of the corresponding quantum system [18, 19].

In contrast to freely tunable parameters, such as external magnetic and electric fields, interactions between particles are inevitably present in any realistic physical

*Electronic address: erasanen@jyu.fi system. While single-particle billiards have been studied thoroughly for many years now, billiards of interacting particles are still a relatively young field. Classical billiards for two interacting particles have been studied using various models, e.g., Coulomb-like interactions in a one-dimensional box [20] and in an isotropic [21] and anisotropic harmonic oscillator [22], as well as applying hard-sphere contact interaction in a rectangular 23. and a mushroom-shaped box [24]. The statistical mechanics of such systems has also been extensively studied recently [25]. Quantum-mechanically, interactioninduced chaos has been studied in a two-electron quantum dot 21, 22, 26], and, very recently, also in the framework of time-dependent density-functional theory [27, 28] - an approach that might enable examination of quantum chaos in systems containing a large number of interacting particles.

Single-particle billiards have traditionally been studied by either reducing the dynamics of the system to a bouncing map (for magnetic single-particle billiards, see, e.g., [29]), or by investigating the infinitesimal variations of the trajectories using the method of Jacobi fields [30, 31]. In an interacting billiard, however, the trajectory of a particle between successive bounces is not known in advance, as its motion is coupled to the motion of all other particles. The locations of the bounces at the wall are not given by simple geometric considerations anymore, and thus the methods used to study single-particle systems do not carry over in a straightforward way.

In this paper, we present a classical molecular dynamics scheme that allows to calculate the trajectories of interacting particles in an arbitrary $2 \mathrm{D}$ billiard system exposed to a uniform and perpendicular magnetic field. To demonstrate the method, we focus on single- and twoparticle dynamics in rectangular billiards. In the singleparticle case, we present an efficient method to systematically obtain "regular" and "chaotic" regions in phase space, which allows us to monitor the combined effect 
of the magnetic field and the rectangle shape. We find a pattern of increased regularity at integer aspect ratios. In the two-particle case mostly chaotic behavior is found, but also regular orbits at high magnetic fields. The relevance of the method in studying the classical limit of collective effects in many-electron structures is discussed.

\section{METHOD}

\section{A. Propagation of particles}

To calculate the trajectories of charged particles, we use a modified velocity verlet algorithm suited for incorporating arbitrarily strong static homogeneous external magnetic fields [32]. With a magnetic field $\mathbf{B}=(0,0, B)$ pointing in $z$ direction, the acceleration of a charged particle reads

$$
\mathbf{a}(t)=\mathbf{a}^{C}(t)-\Omega \mathbf{e}_{z} \times \mathbf{v}(t),
$$

where $\mathbf{a}^{C}(t)$ is the velocity-independent part of the acceleration depending only on external forces, and $\Omega=$ $q B / m$ is the cyclotron frequency for a particle with charge $q$ and mass $m$. We use Hartree atomic units throughout the paper, such that $\hbar=e=m_{e}=$ $1 /\left(4 \pi \epsilon_{0}\right)=1$ and the velocity of light has the value $c \approx 137.036$. Furthermore, the factor $1 / c$ in the Lorentz force law is absorbed into $B$, such that we have $\Omega=B$ for electrons. Within the modified velocity verlet algorithm presented in Ref. [32], each particle is propagated using the following equations:

$$
\begin{aligned}
& r_{x}(t+\Delta t)=r_{x}(t)+\frac{1}{\Omega}\left[v_{x}(t) \sin (\Omega \Delta t)-v_{y}(t)[\cos (\Omega \Delta t)-1]\right]+ \\
& +\frac{1}{\Omega^{2}}\left[-a_{x}^{C}(t)[\cos (\Omega \Delta t)-1]-a_{y}^{C}(t)[\sin (\Omega \Delta t)-\Omega \Delta t]\right]+\mathcal{O}\left[(\Delta t)^{3}\right] \\
& r_{y}(t+\Delta t)=r_{y}(t)-\frac{1}{\Omega}\left[-v_{y}(t) \sin (\Omega \Delta t)-v_{x}(t)[\cos (\Omega \Delta t)-1]\right]+ \\
& +\frac{1}{\Omega^{2}}\left[-a_{y}^{C}(t)[\cos (\Omega \Delta t)-1]-a_{x}^{C}(t)[-\sin (\Omega \Delta t)+\Omega \Delta t]\right]+\mathcal{O}\left[(\Delta t)^{3}\right] \\
& \mathbf{a}^{C}(t+\Delta t)=\mathbf{a}^{C}\left[r_{1}(t+\Delta t), \ldots, r_{N}(t+\Delta t) ; t+\Delta t\right] \\
& v_{x}(t+\Delta t)=v_{x}(t) \cos (\Omega \Delta t)+v_{y}(t) \sin (\Omega \Delta t)+\frac{1}{\Omega}\left[-a_{y}^{C}(t)[\cos (\Omega \Delta t)-1]+\right. \\
& \left.+a_{x}^{C}(t) \sin (\Omega \Delta t)\right]+\frac{1}{\Omega^{2}}\left[-\frac{a_{x}^{C}(t+\Delta t)-a_{x}^{C}(t)}{\Delta t}[\cos (\Omega \Delta t)-1]-\right. \\
& \left.-\frac{a_{y}^{C}(t+\Delta t)-a_{y}^{C}(t)}{\Delta t}[\sin (\Omega \Delta t)-\Omega \Delta t]\right]+\mathcal{O}\left[(\Delta t)^{3}\right] \\
& v_{y}(t+\Delta t)=v_{y}(t) \cos (\Omega \Delta t)-v_{x}(t) \sin (\Omega \Delta t)-\frac{1}{\Omega}\left[-a_{x}^{C}(t)[\cos (\Omega \Delta t)-1]-\right. \\
& \left.-a_{y}^{C}(t) \sin (\Omega \Delta t)\right]+\frac{1}{\Omega^{2}}\left[-\frac{a_{y}^{C}(t+\Delta t)-a_{y}^{C}(t)}{\Delta t}[\cos (\Omega \Delta t)-1]-\right. \\
& \left.--\frac{a_{x}^{C}(t+\Delta t)-a_{x}^{C}(t)}{\Delta t}[-\sin (\Omega \Delta t)+\Omega \Delta t]\right]+\mathcal{O}\left[(\Delta t)^{3}\right]
\end{aligned}
$$

\section{B. Phase space maps for single-particle billiards}

In a single-particle billiard system, the kinetic energy, and consequently also the velocity $v=\left(v_{x}^{2}+v_{y}^{2}\right)^{1 / 2}$ of the particle, is a constant of motion. The dynamics of the billiard is determined by the boundary conditions (see below) and the relative strength of the magnetic field. The latter quantity is here given by a parameter

$$
\mu=R_{c} / L_{x}
$$


where $R_{c}=v / B$ is the cyclotron radius and $L_{x}$ is the length of one side of the system (here a rectangle). The constant of motion can be used to reduce the four-dimensional phase space $\left(x, y, v_{x}, v_{y}\right)$ to a threedimensional (3D) one, where we have chosen the space spanned by $\left(x, y, v_{x}\right)$. To identify regular and chaotic regions in this phase space, we use the following procedure:

1. We choose a $2 \mathrm{D}$ cross section $\left(x, v_{x}\right)$ through the $3 \mathrm{D}$ phase space and divide it into a number of cells.

2. For one cell, we pick two phase space points in the cell that are very close to each other, but not identical up to the numerical precision.

3. We follow the trajectories through these two points for a certain propagation distance $s_{\text {tot }}$ using Eqs. (2)-(6), and record all cells in the cross section through which they pass.

4. After having propagated for a distance $s_{\text {tot }}$, we calculate the distance between the points in phase space, which is a measure of the "regularity" of the trajectory. We save this distance to all cells we have passed. If a cell has already been passed by a previous run, we take the maximum of the distances.

5. We start over from point (2) by picking another cell that has not yet been traversed by a trajectory, and repeat the whole process until all cells have been hit by a trajectory at least once.

6. We then plot the distances stored in the cells of our 2D cross section as a color-coded "matrix plot". In the following, these plots will be called "phasespace maps". Small numbers correspond to "regular" phase space cells, large numbers to "chaotic" cells (see below for details).

The algorithm can be efficiently parallelized, because trajectories originating from different cells can be propagated independent of each other. Our code uses the Message Passing Interface (MPI) and a master/slave paradigm. The master process keeps track of the phase space map and distributes free cells, i.e., cells that have not yet been hit by any trajectory) to the workers. The workers perform the propagation of the trajectories and communicate the traversed cells and the phase space distance after the propagation distance $s_{\text {tot }}$ back to the server.

\section{RESULTS}

\section{A. Single particle}

We demonstrate our computational scheme by considering rectangular billiards with side lengths $L_{x}=1$ (fixed) and $L_{y}=\beta L_{x}$ (varied), where $\beta$ is the aspect ratio. The strength of the external magnetic field has been
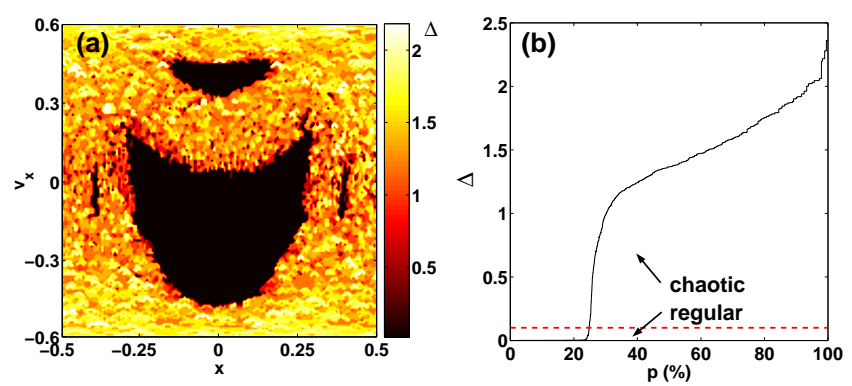

FIG. 1: (color online). (a) Phase space map for a rectangular billiard with aspect ratio $\beta=2$. The color map indicates the phase-space distance $\Delta$ between two orbits having a small initial perturbation. (b) Ordered phase-space distances for all the cells plotted in (a). The dashed line shows the threshold $(\Delta=0.1)$ between chaotic and regular motion.

fixed to $B=1$, so that the cyclotron radius $R_{c}=v / B$ is determined by varying the velocity of the particle. In the single-particle case, we focus on the dynamics of the system as a function of $\beta$ and $\mu=R_{c} / L_{x}$. In both of the limits $\mu \rightarrow 0$ and $\mu \rightarrow \infty$ the motion is regular, the former corresponding to infinitely many circular orbits (cf. Landau-level condensation in confined quantum systems) and the latter corresponding to linear motion at zero field, which is always regular in rectangular billiards. At $0<\mu<\infty$ the dynamics is generally mixed except at particular values of $\mu$ when the system is completely chaotic 13].

Fig. 1(a) shows an example of a phase space map calculated for the parameters $\beta=2$ and $\mu=0.6$. The scheme described in Sec. IIB has been used to calculate the figure. The cross section through the phase space has been partitioned into 150 cells in each direction $(x$ and $\left.v_{x}\right)$. The color scale indicates the phase-space distance $\Delta$ after propagating the trajectories by a distance of $s_{\text {tot }}=60 L_{x}$.

We find distinct areas of regularity associated with KAM (Kolmogorov-Arnold-Moser) islands [1]. Figure 1(b) shows the phase-space distances of all cells sorted in ascending order. The sharp onset of the curve indicates a distinct separation between regular and chaotic motion. To consistently determine this separation, we choose a threshold of $\Delta=0.1$ shown in the figure as a dashed line. Thereby, this particular system is regular by a fraction of $25 \%$. We presume that by using a very high resolution it should be possible to determine and categorize phase-space cells corresponding to weak chaos [33]. This topic is, however, beyond the scope of this work and left for future research.

In Fig. 2 we show the proportions of regularity, estimated as shown in the example in Fig. 1 for square $(\beta=1)$ billiards as a function of $\mu$. We find excellent agreement with the result of Berglund and Kunz [13] that has been calculated using an exact method. This confirms the accuracy of the proposed scheme up to strongly curvilinear motion, i.e., small values of $\mu$. Hence, we ex- 


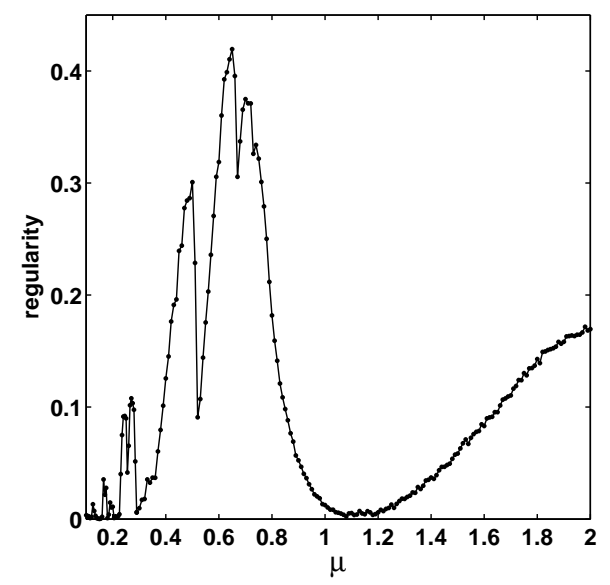

FIG. 2: Proportions of regularity in square billiards as a function of $\mu=R_{c} / L_{x}$, i.e., the ratio between the cyclotron radius and the side length.

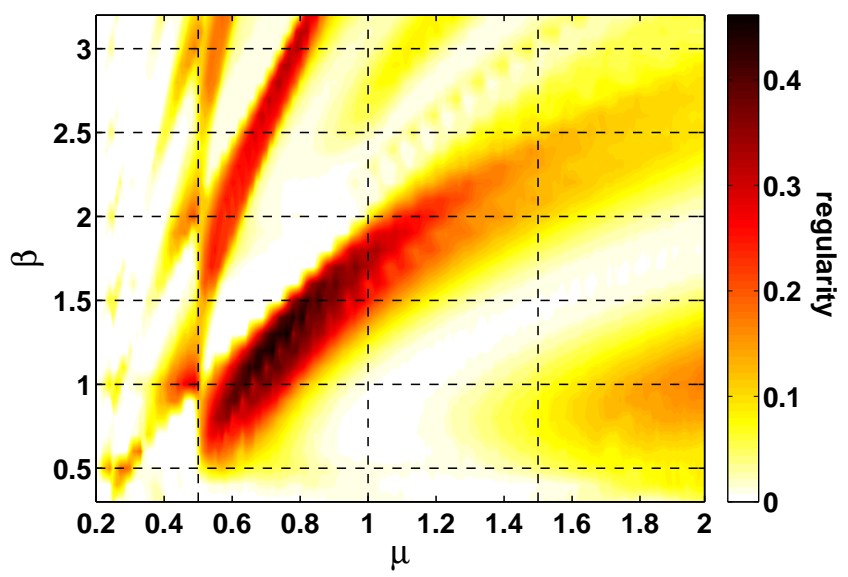

FIG. 3: (color online) Proportions of regularity in rectangular billiards as a function of $\mu$ and the aspect ratio $\beta$.

pect the method to be reliable also in more complicated systems with many particles and/or different boundaries.

To assess the effect of the billiard shape onto the dynamics, we have calculated the proportions of regularity as a function of both $\mu$ and the aspect ratio $\beta$. The result is shown in Fig. 3 for $0.3<\beta<3.2$ in steps of 0.1 and for $0.2<\mu<2$ in steps of 0.01 . Fig. 3 required the calculation of 5430 phase space maps, each consisting of 22500 cells, thereby demonstrating the numerical efficiency of the scheme. Note that Fig. [3 is not symmetric around $\beta=1$, because we have varied $L_{y}=\beta$ and thus the system area is not kept constant.

We find several islands of increased regularity centered at $\beta=n / 2$ with $n=1,2,3, \ldots$. Overall, the "most regular" case is the square billiard $(\beta=1)$, as expected. A more detailed analysis of the regularity patterns and their connections to the periodic orbits will be performed elsewhere.

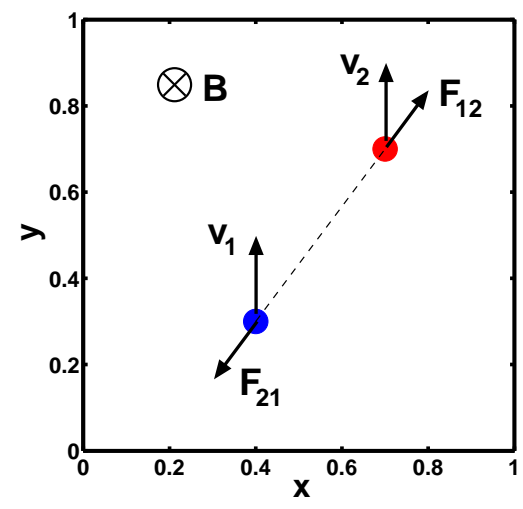

FIG. 4: (color online). Initial configuration of the calculations for two Coulomb-interacting particles in square billiards subjected to a perpendicular magnetic field.

\section{B. Two particles}

We now turn to the dynamics of two particles interacting via Coulomb repulsion in a square well $(\beta=1)$. Now the velocities (and thus also the cyclotron radii) are no longer constants of motion. The phase space is eight-dimensional, and instead of the phase space map described in section IIB, we calculate so-called bouncing maps by recording the values $\left(x, v_{x}\right)$ corresponding to the bounces of one of the particles on the lower boundary $(y=0)$ of the system.

We investigate the dynamics with different values for the ratio

$$
\gamma=\frac{E_{\mathrm{k}}(t=0)}{E_{\mathrm{p}}(t=0)}
$$

for the initial configuration, where $E_{p}=\left|\mathbf{r}-\mathbf{r}^{\prime}\right|^{-1} / 2$ is the Coulomb potential energy and $E_{\mathrm{k}}=v^{2} / 2$ is the kinetic energy. The quantity $\gamma$ essentially determines how "strongly interacting" the system is, as it fixes the average ratio of $E_{k}(t)$ and $E_{p}(t)$ for the full time-dependent system through the initial energy components. In physical applications this ratio could be varied by changing either the particle density or the system size. A wellknown example of the limit where the potential energy dominates is the Wigner crystal [34] forming in the electron gas at low densities.

In the following examples we have fixed the initial positions of the particles to $\left(x_{1}, y_{1}\right)=(2 / 5,3 / 10)$ and $\left(x_{2}, y_{2}\right)=(7 / 10,7 / 10)$. The initial Coulomb energy in this case is $E_{p}(t=0)=2$. After fixing $\gamma$ in Eq. (8), the initial kinetic energy $E_{k}(t=0)$ is distributed equally to both particles, and the initial velocities $\mathbf{v}_{1}=\mathbf{v}_{2}=$ $\left(0, \sqrt{E_{k}}\right)$ point in the $y$ direction. The initial configura-

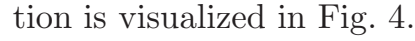

The remaining parameter to be fixed is $\mu(t=0)$ defined in Eq. (77). Note that again we fix only the initial condition, and in the time-dependent run, the values of $\mu$ for both particles vary due to changes in the velocities. 



FIG. 5: (color online). Upper panel: Classical trajectories for two relatively weakly interacting particles indicated by blue (black) and red (gray) colors in square billiards. The magnetic field is zero. Lower panel: Bouncing map for the particle with the blue (black) trajectory in the upper panel.

Since the initial velocity is determined through $\gamma$, we fix $\mu(t=0)$ through $B$ in contrast with the single-particle case where we always had $B=1$.

First, we set $\gamma=30$ and the magnetic field to zero $(\mu \rightarrow \infty)$ and propagate sufficiently long to obtain a bouncing map with a large number of points. Figure 5 shows the trajectories of the particles up to $t=5$ (upper panel) and the bouncing map up to $t=3 \times 10^{4}$ (lower panel). The number of bounces is $\sim 7.8 \times 10^{5}$. Apart from a few exceptions, the particles remain separated in the left and right parts of the system due to the Coulomb repulsion. However, as the interaction is relatively weak, both particles move in the $y$ direction, almost undisturbed from their initial conditions. Close to the left and right boundaries, where the interaction is weakest, the dynamics is most regular. This can be seen in the trajectories, which are almost straight lines in that regime. In addition, the bouncing map shows regular curvilinear albeit blurry zones (see the inset in the lower panel of Fig. (5). These features may be designated as quasi-regular motion in the system [33].
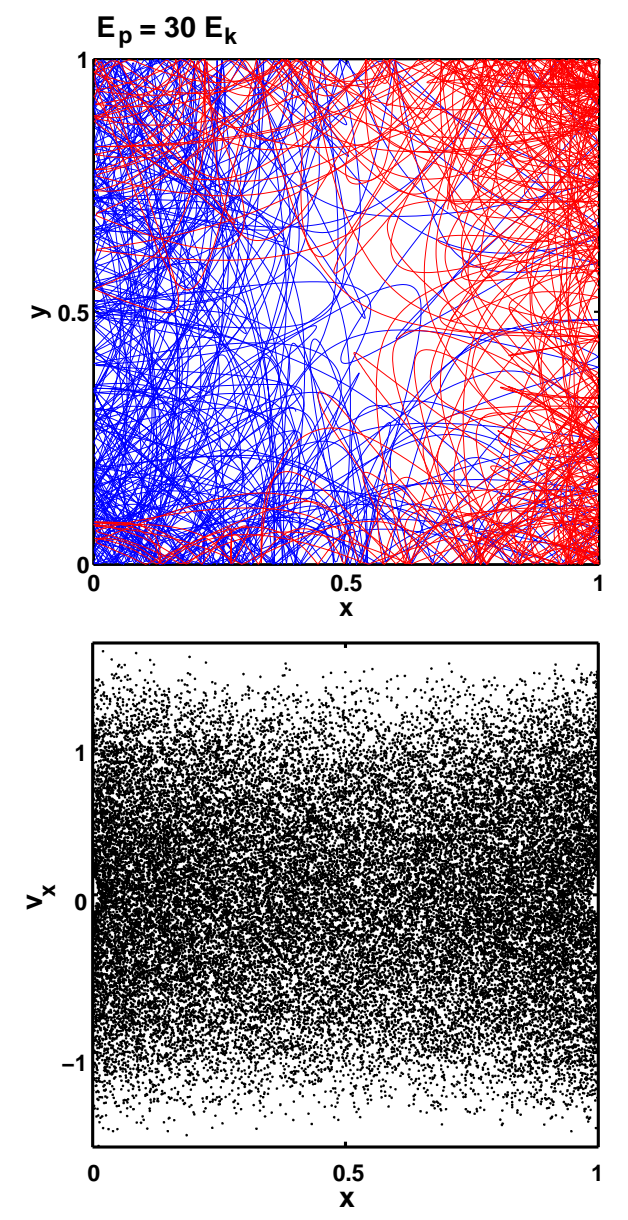

FIG. 6: (color online). Same as Fig. 5 but for relatively strongly interacting particles.

In the following example we keep the magnetic field at zero but increase the relative amount of interaction energy such that $\gamma=1 / 30$. The trajectories and bouncing map are shown in Fig. 6. Here, the dynamics is very different from the weakly interacting case. Both particles occupy the whole area of the system, but due to their strong repulsion, the corners are considerably more occupied than the central region, which is characterized by "scattering" trajectories of high curvature. The bouncing map in the lower panel is completely chaotic. Increasing the interaction even further would enable to study classical Wigner crystallization 34 in a dynamic picture. In the present system, for example, the Wigner crystal would consist of two diagonal configurations summed up to a four-point crystal.

Finally, we consider two systems with $\gamma=2$, where the magnetic field is set to values corresponding to $\mu(t=$ $0)=1 / 4$ and $\mu(t=0)=1 / 32$, respectively. The trajectories are plotted in Fig. 7 In the first case (a) the system seems to be fully chaotic, whereas the latter configuration (b) leads to regular isolated orbits forming a ring-like structure. In this case, the "interaction axis" (i.e., the dashed line in Fig. (4) performs a circular mo- 

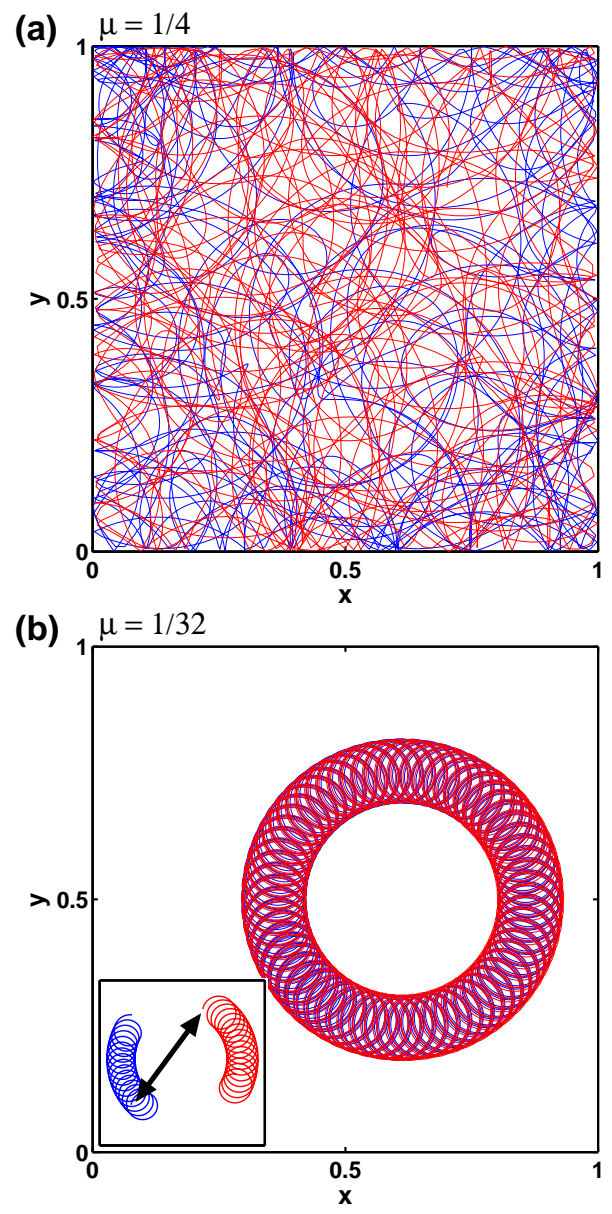

FIG. 7: (color online). Classical trajectories for two interacting particles at two different magnetic fields corresponding to $\mu=1 / 4$ and $\mu=1 / 32$, respectively.

tion that is superimposed by strongly confined cyclotron motions at the opposite ends of the axis. The characteristics of this motion are further illustrated in the inset of Fig. Z(b), which shows the trajectories soon after the beginning of the time propagation. The - at a first glance - counterintuitive result that a repulsive interaction leads to bound motion can be understood by considering the combined effect of Coulomb repulsion and the strong magnetic confinement through the cyclotron motion. When the particles increase their relative distance, the gain in kinetic energy (at the expense of Coulomb energy) results in an increased radius of the cyclotron motion. The different curvature of the trajectory on the different sides of the "circle" gives rise to a bent cycloidal motion which can, for the right choice of the parameters, lead to a bound motion as depicted in Fig. 7(b).

The above results on the classical dynamics in magnetic fields suggest to study the relation to the corresponding quantum mechanical situation in semiconductor quantum dots. In fact, interesting vortex patterns and edge localization have been found in rectangular many-electron quantum dots at high magnetic fields [35]. Such patterns may exist - in a statistical picture - also in a classical system. A particularly interesting case would be the quantum-mechanical analog of the bound motion shown in Fig. 7(b). Moreover, our scheme would allow to study the effects of interactions on the classical limit of electron transport in billiard arrays [12].

\section{SUMMARY}

We have introduced a computational scheme based on molecular dynamics to study classical billiards of interacting particles in external magnetic fields. The accuracy and efficiency of the method has been demonstrated in rectangular billiards. We have found excellent agreement with numerically exact method in single-particle square billiards as a function of the magnetic field. Changing the aspect ratio $\beta$ of the rectangle leads to islands of increased regularity at $\beta=n / 2$ with $n=1,2,3, \ldots$ In square billiards of two interacting particles we have found signatures of quasiperiodic orbits at weak interactions, and localization at strong interactions. Large magnetic fields may lead to regular patterns also for interacting particles. The scheme opens up the path to study the classical limit of realistic many-particle systems related with, e.g., electronic transport experiments in mesoscopic structures.

\section{Acknowledgments}

We thank Roland Brunner for useful disdcussions. This work was supported by the Academy of Finland.
[1] M. C. Gutzwiller, Chaos in Classical and Quantum Mechanics (Springer Verlag, New York, 1990).

[2] H.-J. Stockmann, Quantum Chaos: An Introduction (Cambridge University Press, Cambridge, 2000).

[3] K. Nakamura and T. Harayama, Quantum Chaos and Quantum Dots (Oxford University Press, Oxford, 2004).

[4] For reviews, see L. P. Kouwenhoven, D. G. Austing, and S. Tarucha, Rep. Prog. Phys. 64, 701 (2001); S. M. Reimann and M. Manninen, Rev. Mod. Phys. 74, 1283
(2002).

[5] R. Hanson, L. P Kouwenhoven, J. R. Petta, S. Tarucha, and L. M. K. Vandersypen, Rev. Mod. Phys. 79, 1217 (2007).

[6] M. V. Berry, M. Tabor, Proc. Royal Soc. London A Mat, 356, 375 (1977).

[7] O. Bohigas, M. J. Giannoni, C. Schmit, Phys. Rev. Lett. 52, 1 (1984).

[8] M. V. Berry, M. Robnik, J. of Phys. A: Math. Gen. 17, 
2413 (1984).

[9] H. Makino, T. Harayama, Y. Aizawa, Phys. Rev. E 59, 4026 (1999).

[10] H. Makino, T. Harayama, Y. Aizawa, Phys. Rev. E 63, 056203 (2001).

[11] M. Robnik and M. V. Berry, J. Phys. A 18, 1361 (1985).

[12] R. Brunner, R. Meisels, F. Kuchar, R. Akis, D. K. Ferry, and J. P. Bird, Phys. Rev. Lett. 98, 204101 (2007).

[13] N. Berglund and H. Kunz, J. Stat. Phys. 83, 81 (1996).

[14] M. Novaes and M. A. M. deAguiar, Phys. Rev. E 70, 045201(R) (2004).

[15] G. Date, S. R. Jain, and M. V. N. Murthy, Phys. Rev. E 51, 198 (1995).

[16] R. Narevich, R. E. Prange, and O. Zaitsev, Phys. Rev. E 62, 2046 (2000).

[17] L. Christensson, H. Linke, P. Omling, P. E. Lindelof, I. V. Zozoulenko, and K.-F. Berggren, Phys. Rev. B 57, 12306 (1998).

[18] M. V. Berry, M. Robnik, J. of Phys. A: Math. Gen. 19, 649 (1986).

[19] M. Robnik, M. V. Berry, J. of Phys. A: Math. Gen. 19, 669 (1986).

[20] L. Meza-Montes and S. E. Ulloa Phys. Rev. E 55, R6319 (1997).

[21] S. Radionov, S. Åberg, and T. Guhr, Phys. Rev. E 70, 036207 (2004).

[22] P. S. Drouvelis, P. Schmelcher, and F. K. Diakonos, Europhys. Lett. 64, 232 (2003).

[23] A. Awazu, Phys. Rev. E 63, 032102 (2001).
[24] S. Lansel, M. A. Porter, and L. A. Bunimovich, Chaos 16, 013129 (2006).

[25] See, e.g., S.-H. Suh and S.-C. Kim, Phys. Rev. E 69, 026111 (2004); M. Uranagase and T. Munakata, Phys. Rev. E 74, 066101 (2006); I. Urrutia, J. Stat. Phys. 131, 597 (2008).

[26] A. J. Fendrik, M. J. Sánchez, and P. I. Tamborenea, Phys. Rev. B 63, 115313 (2001).

[27] A. Wasserman, N. T. Maitra, and E. J. Heller, Phys. Rev. A 77, 042503 (2008).

[28] For a review on time-dependent density-functional theory, see, e.g., Time-Dependent Density Functional Theory, Lecture Notes in Physics, edited by M. A. L. Marques, C. A. Ullrich, F. Nogueira, A. Rubio, K. Burke, and E. K. U. Gross (Springer, Berlin, 2006).

[29] O. Meplan, F. Brut, C. Gignoux, J. of Phys. A: Math. Gen. 26, 237 (1993).

[30] Z. Vörös, T. Tasnadi, J. Cserti, P. Pollner, Phys. Rev. E 67, 065202(R) (2003).

[31] T. Tasnadi, Commun. Math. Phys 187, 597 (1997).

[32] Q. Spreiter and M. Walter, J. Comp. Phys. 152, 102 (1999)

[33] G. M. Zaslavsky, R. Z. Sagdeev, D. A. Usikov, and A.A. Chernikov, Weak Chaos and Quasiregular Patterns (Cambridge University Press, Cambridge, 1991).

[34] E. P. Wigner, Phys. Rev. 46, 1002 (1934).

[35] E. Räsänen, A. Harju, M. J. Puska, and R. M. Nieminen, Phys. Rev. B 69, 165309 (2004). 